104 


\title{
CONSUMOS ENERGÉTICOS DE GAS NATURAL Y ELECTRICIDAD EN EDIFICIOS ESCOLARES DEL ÁREA METROPOLITANA DE SAN JUAN, ARGENTINA. ANÁLISIS ESTADÍSTICO EN FUNCIÓN DE VARIABLES ARQUITECTÓNICAS
}

\author{
THE NATURAL GAS AND ELECTRIC ENERGY CONSUMPTION OF \\ SCHOOL BUILDINGS IN THE METROPOLITAN AREA OF SAN JUAN, \\ ARGENTINA: A STATISTICAL ANALYSIS BASED ON ARCHITECTURAL \\ VARIABLES.
}

MARÍA GUILLERMINA RE

Magister Arquitecta. Estudiante de Doctorado en Arquitectura, Universidad de Mendoza Universidad Nacional de San Juan (UNSJ) San Juan, Argentina https://orcidorg/0000-0002-3109-7138

guillerminare@gmail.com
CELINA FILIPPÍN

Doctora en Ciencias, Especialidad Energías Renovables Consejo Nacional de Investigaciones Científicas y Tecnológicas (CONI(ET), Santa Rosa. La Pampa, Argentina https://orcid.org/0000-0002-0521-6180 cfilippin@cpenet.com.ar

\author{
IRENE BLASCO LUCAS \\ Doctora Arquitecta \\ Instituto Regional de Planeamiento y Hábitat (IRPHA). \\ Facultad de Arquitectura, Urbanismo Diseño (FAUD) \\ Universidad Nacional de San Juan (UNSJ)San Juan, Argentina \\ https://orcid.org/0000-0002-1326-895X \\ iblasco06@gmail.com
}

\section{RESUMEN}

El presente trabajo tiene por objetivo indagar sobre los consumos energéticos de 17 escuelas localizadas en el Área Metropolitana de San Juan. Se analizan aquí los consumos por año y por unidad de superficie para obtener una lectura general de la situación actual. Además, se realizan análisis estadísticos con el fin de comprender las posibles relaciones entre los consumos energéticos y dos variables arquitectónicas que caracterizan el diseño y la tecnología constructiva: Área vidriada al Norte y K global. Los resultados indican que la energía con mayor participación anual relativa es la eléctrica (55\%), mientras que el gas natural lo es durante la estación fría (72\%), asociado al abastecimiento de energía en calefacción. Respecto al consumo por tipo de energía y por unidad de superficie cubierta, los valores son de $26,24 \mathrm{kWh} / \mathrm{m}^{2}$. año para la electricidad y de $21,80 \mathrm{kWh} / \mathrm{m}^{2}$. año para el gas natural. El desarrollo de la investigación permite concluir que existe una importante asociación entre la envolvente edilicia, tanto opaca como transparente, y el consumo de energía, con lo cual se reconoce la potencialidad de mejoramiento de la envolvente, en búsqueda de una optimización energética.

Palabras clave

arquitectura, energía, escuelas

ABSTRACT

The present work aims to investigate the energy consumption of 17 schools located in the Metropolitan Area of San Juan, Argentina. To obtain a general overview of the current situation, consumption per year and per unit area were analyzed. In addition, statistical analyses were carried out in order to understand possible relationships between energy consumption and two architectural variables that characterize design and construction technology: glazed area to the north and global U-value. The results indicate that electricity is the energy with the greatest relative annual use (55\%), while natural gas predominates during the cold season (72\%), as associated with heating energy supply. Regarding consumption by type of energy and per unit area covered, the values were $26.24 \mathrm{kWh} /$

$\mathrm{m}^{2}$.year for electricity and $21.80 \mathrm{kWh} / \mathrm{m}^{2}$.year for natural gas. Through the research it was possible to conclude that there is an important association between the building envelope, both opaque and transparent, and energy consumption, thereby recognizing the potential to improve the envelope for energy optimization. 


\section{INTRODUCCIÓN}

El consumo energético de edificios es un tema a considerar en momentos de escasez de recursos no renovables, como ocurre actualmente a nivel mundial. Según los datos publicados por la Secretaría de Energía de la Nación (2012), la participación de los edificios del sector residencial, comercial y público en el consumo total de energía es del 31\%; mientras que, si se considera solo el consumo de electricidad, este es del $55 \%$, y el de gas por red, del $50 \%$. El alto consumo registrado requiere de la implementación de planes de optimización de eficiencia energética, con el fin de disminuir la demanda.

La falta de conocimiento sobre los factores que determinan el uso de la energía en edificios representa una dificultad para lograr la eficiencia energética de los mismos (Yoshino, Hong y Nord, 2017). Las escuelas son edificios públicos que poseen particularidades en su diseño, construcción y funcionamiento; es por ello que el análisis de consumos en tipologías escolares a partir de datos empíricos de auditorías energéticas es un tema que ha interesado a numerosos grupos de investigación en diferentes partes del mundo (Rospi et al, 2015; Trebilcock et al., 2016; Raatikainen et al., 2016; Mohamed y Mohamed, 2017; Droutsaa et al., 2017). En Daegu, Corea del Sur, un estudio llevado a cabo sobre 10 escuelas primarias, analiza los consumos históricos, por año y por unidad de superficie, arribando a las siguientes conclusiones: que la energía eléctrica es la más utilizada, seguida del gas; que el consumo de energía por unidad de superficie áulica fue de $289 \mathrm{kWh} / \mathrm{m}^{2}$. año de electricidad y $90 \mathrm{kWh} / \mathrm{m}^{2}$.año de gas, durante el año 2010; y que, en términos de los usos, se consume más energía en calefacción, seguido de enfriamiento e iluminación (Woo, Guk y Hwa, 2012).

En Argentina también se han desarrollado importantes contribuciones desde el ámbito científico en la temática de escuelas (Filippín, 2005; Melchiori, San Juan y Díscoli, 2014; Boutet, Hernández y Jacobo, 2016; Ledesma et al., 2016; Walter et al., 2016; Giuliano y Garzón, 2017; Mazzocco, Filippín y Flores Larsen, 2017) y en el manejo de estudios estadísticos como una herramienta utilizada para la caracterización térmica y energética en edificios (Czajkowski, 2009; Muñoz, Marino y Thomas, 2016; Boutet, 2017).

En San Juan, diversas investigaciones han permitido agrupar las escuelas del Área Metropolitana en tipologías edilicias, como también conocer el comportamiento energético, los niveles de confort higrotérmico y la vulnerabilidad sísmica de distintos casos pertenecientes a períodos históricos representativos (Michaux, 2014; Ré, Blasco Lucas y Fillipín, 2016; 2017; Yacante et al., 2017). El presente trabajo tiene por objetivo aportar datos empíricos respecto a los consumos de gas y electricidad registrados en escuelas $y$, asimismo, identificar las relaciones existentes con variables arquitectónicas que definen la envolvente edilicia.

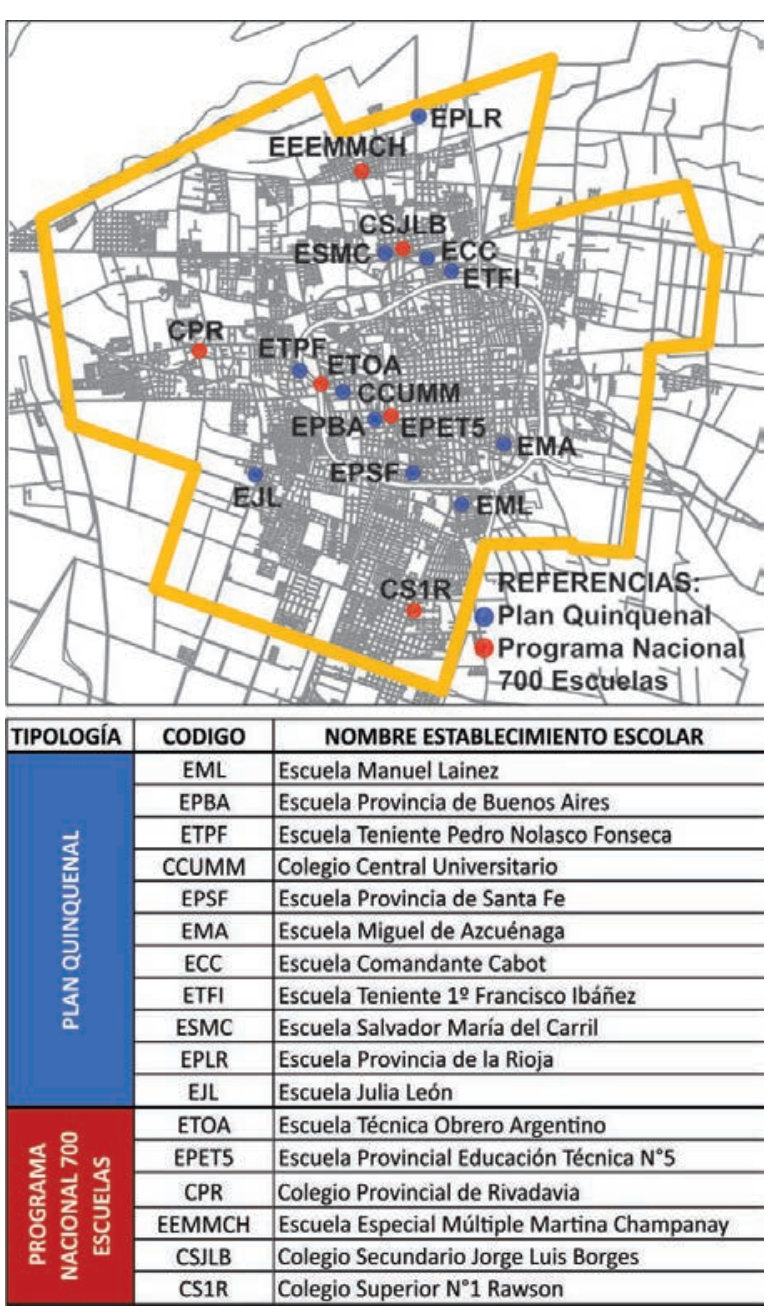

Figura 1. Área Metropolitana de San Juan. Ubicación de los establecimientos escolares. Fuente: Elaboración de las autoras.

\section{UNIDADES DE ANÁLISIS}

Se considera como población de estudio a las escuelas de dos tipologías arquitectónicas del Área Metropolitana de San Juan (AMSJ) que registran los mayores índices de construcción de infraestructura escolar de gestión pública. Una de ellas corresponde a la época de la reconstrucción de la ciudad, post terremoto de 1944, con el prototipo ideado en el marco del Plan Quinquenal (PQ); la otra tipología concierne al Programa Nacional 700 Escuelas (PN700) desarrollado entre los años 2004 a 2008. Para el presente artículo se toma una muestra representativa (Sierra Bravo, 1991) compuesta por 17 Unidades de Análisis (UA), la cual corresponde a un $85 \%$ de la población en estudio y estuvo definida al considerar todos aquellos establecimientos que cuentan con el servicio de energía eléctrica y gas natural por red. En la Figura 1, se puede observar la localización de los edificios escolares junto con una tabla de referencias que los identifica; se establece un código respecto al nombre y un color de acuerdo a la tipología edilicia a la que pertenecen: en azul los del PQ (11 UA) y en rojo los del PN700 (6 UA). 


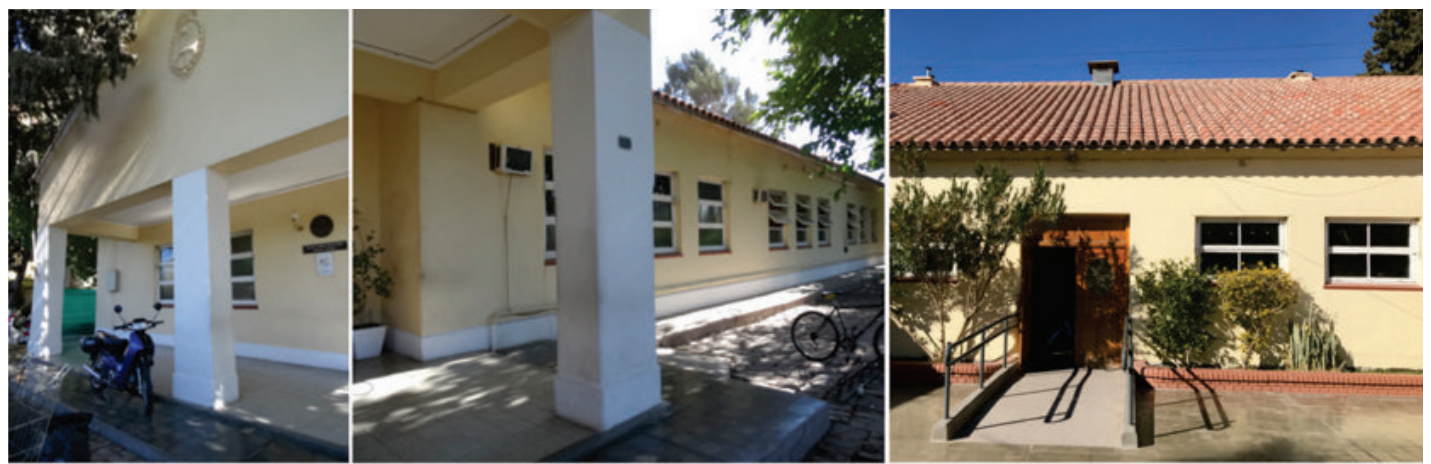

COLEGIO CENTRAL UNIVERSITARIO MARIANO MORENO (CCUMM)

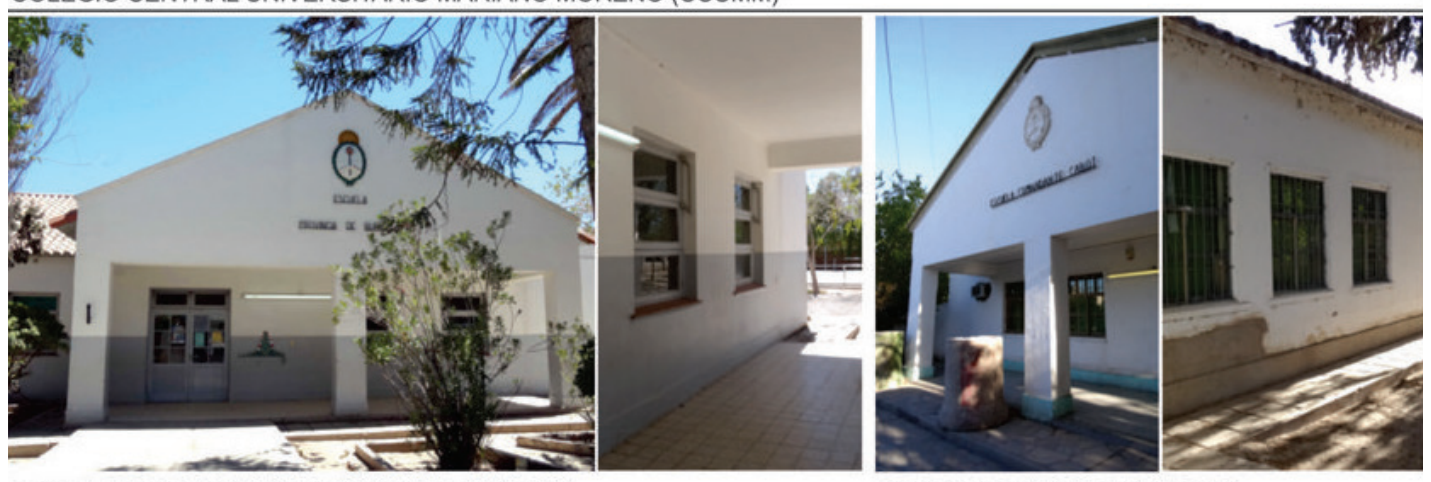

ESCUELA PROVINCIA DE BUENOS AIRES (EPBA)

E. COMANDANTE CABOT (ECC)

Figura 2. Casos de la tipología PQ. Fotografías: Guillermina Ré.

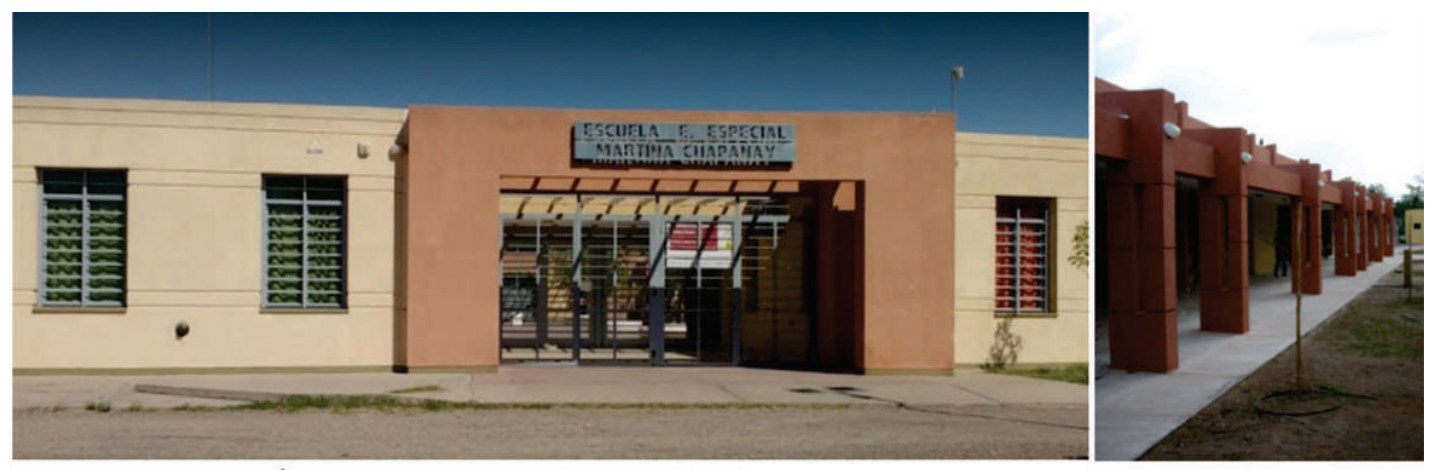

ESCUELA ESPECIAL MÚLTIPLE MARTINA CHAPANAY (EEMMCH)

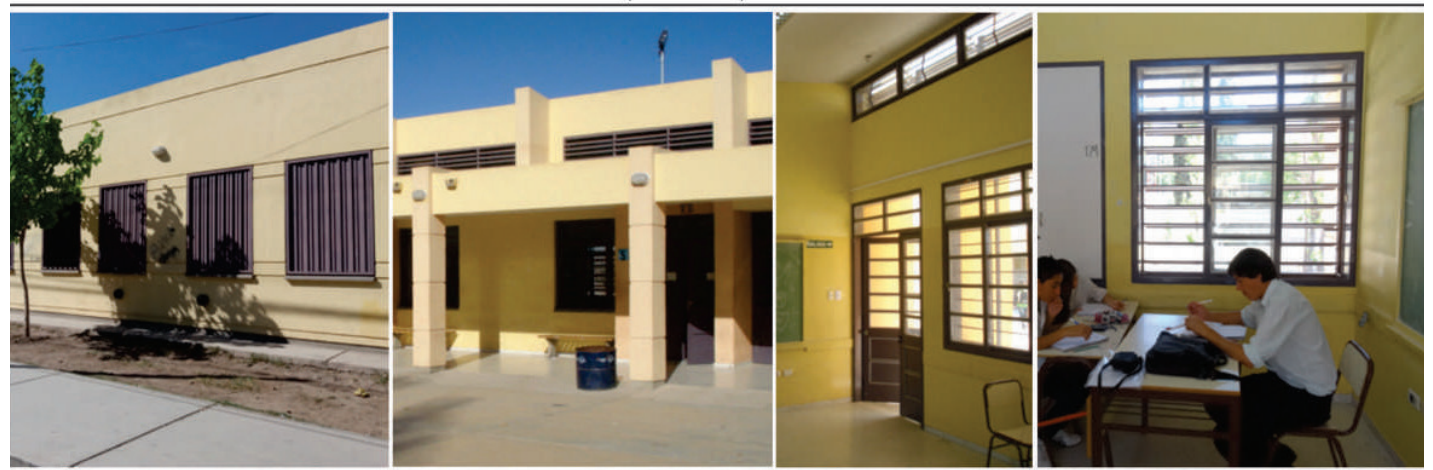

COLEGIO PROVINCIAL DE RIVADAVIA (CPR)

Figura 3. Casos de la tipología PN700. Fotografías EEMMCH: Programa Nacional 700 Escuelas (http://www.700escuelas.gov.ar) y Raúl Alfonzo. Fotografías CPR: Guillermina Ré. 
Las escuelas del PQ poseen una antigüedad superior a 50 años y se encuentran en pleno funcionamiento, algunas con mejor estado de conservación que otras (Figura 2). La mayoría poseen ampliaciones realizadas con diferente tecnología constructiva que la utilizada en la tipología original (Ré, Blasco y Filippín, 2017). Las infraestructuras del PN700 (Figura 3) se encuentran caracterizadas por su morfología y materialización de la envolvente (Ré, Blasco y Filippín, 2016) y no por una similar organización funcional, como ocurre con las del PQ. En cuanto a los equipos para climatización, todas las UA utilizan estufas y/o pantallas a gas para calefaccionar los ambientes interiores, sin embargo, algunas escuelas poseen estufas eléctricas o caloventores auxiliares en los sectores administrativos. Para refrigeración, solo las escuelas ETPF y CCUMM poseen aires acondicionados (AA) en las aulas.

\section{METODOLOGÍA}

Losconsumosenergéticosfueron solicitados a las empresas proveedoras del servicio de gas natural y electricidad, y corresponden a un período variable de diez años (2006 a 2015) para las escuelas con mayor antigüedad (PQ); y desde el primer año de funcionamiento, para aquellas de reciente construcción (PN700). Los datos de consumos de gas, que originalmente estaban en $\mathrm{m}^{3}$, se convirtieron a kWh para posibilitar el análisis junto a los de electricidad, utilizando un factor de $8,33 \mathrm{kWh} / \mathrm{m}^{3}$ (Blasco Lucas, 2013).

Simultáneamente, se computan las distintas áreas de uso. Se distinguen las superficies cubiertas de las climatizadas ya que, a diferencia de lo que ocurre en viviendas u otros edificios públicos, en las escuelas existen importantes áreas cubiertas sin climatización artificial con equipos de calefacción y/o refrescamiento, por ejemplo: sanitarios, depósitos, circulaciones y salón de usos múltiples (SUM), en algunos casos.

En una primera instancia del estudio que, como se ha indicado, tiene por finalidad conocer la situación general respecto a los consumos energéticos en edificios escolares, se trabaja con la totalidad de los datos obtenidos. En una segunda etapa, la muestra se reduce a los años 2014 y 2015, que son aquellos con información de consumos eléctricos y de gas para las 17 unidades de análisis.

Para definir las variables arquitectónicas a estudiar, se realiza previamente una matriz de correlación de distintas variables de diseño que caracterizaran la morfología, tecnología, funcionalidad y uso de los edificios escolares, y los consumos anuales promedio de los años 2014 y 2015, diferenciados en: total, estacional y por fuente de energía. A partir de ella, se seleccionan dos variables que definen a la envolvente edilicia exterior y están estrechamente ligadas a un diseño que apunta al ahorro energético: Superficie vidriada al Norte $\left(\mathrm{m}^{2}\right)$ y K global $\left(\mathrm{W} / \mathrm{m}^{2 \circ} \mathrm{C}\right)$.
En cuanto al análisis estadístico, se utiliza el software Statgraphics. Se realiza un análisis de regresión lineal simple con el fin de estimar los valores de la variable dependiente (consumo de energía), a partir de la obtención de la función lineal. La anotación matemática de la ecuación es: $y=a+b^{\star} x$. Se valora, entonces, la bondad de ajuste de los datos al modelo de regresión lineal simple a través de los indicadores estadísticos: I) Coeficiente de Correlación Lineal Simple (r), que mide el grado de asociación lineal entre dos variables que oscila entre 1 (fuerte asociación lineal positiva: a medida que aumenten los valores de una variable aumentarán los de la otra) y -1 (fuerte asociación lineal negativa: a medida que aumenten los valores de una variable disminuyen los de la otra); y II) Valor P: un valor menor a 0,05 indica una asociación estadísticamente significativa con un nivel de confianza del $95 \%$.

Para calcular la transmitancia térmica ( $K$, en la denominación argentina; $U$ en el ámbito internacional) de los elementos que componen la envolvente edilicia, se utiliza el programa desarrollado por Gonzalo et al. (2000). Los valores del K global, se obtienen con las planillas programadas KG-MOD (Blasco Lucas, 2013), que aplican los procedimientos sugeridos en las Normas IRAM 11601, 11604 y 11605.

\section{CONSUMOS DE ENERGÍA ELÉCTRICA Y GAS NATURAL}

En primer término, se analizan los consumos energéticos anuales promedio de las escuelas, que permitan una lectura general de la temática en estudio. La Figura 4 muestra que los valores oscilan entre $32.378,93 \mathrm{kWh}$ (ETPF) y 190.461,83 kWh (CCUMM), para las escuelas de tipología $P Q$, y entre $83.899,32 \mathrm{kWh}$ (CPR) y $158.545,82$ $\mathrm{kWh}(\mathrm{EEMMCH})$, para las infraestructuras del PN700.

En relación a los consumos por unidad de superficie cubierta y climatizada (Figura 5), se observa que la escuela con menor consumo es la ETPF, con valores anuales de $23,56 \mathrm{kWh} / \mathrm{m}^{2}$ respecto a la cubierta y $39,22 \mathrm{kWh} / \mathrm{m}^{2}$ a la climatizada. Los mayores consumos los registra la EEMMCH de tipología PN700, con valores de 111,82 kWh/m² y $149,44 \mathrm{kWh} / \mathrm{m}^{2}$, respectivamente, seguida del CCUMM (PQ), con consumos anuales de 80,33 $\mathrm{kWh} / \mathrm{m}^{2}$, según el área cubierta y $123,92 \mathrm{kWh} / \mathrm{m}^{2}$ del área climatizada.

En complemento, se estudia la variación temporal de los consumos energéticos anuales por 


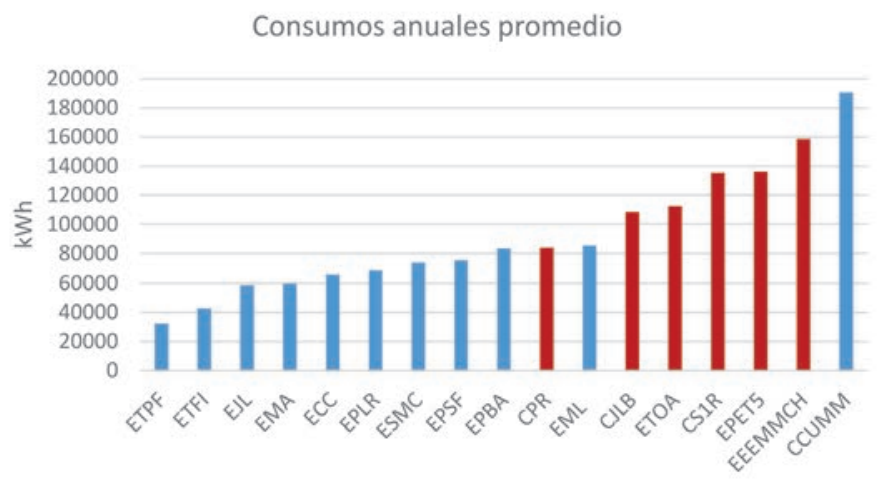

Códigos de cada escuela, diferenciadas según tipología edilicia.

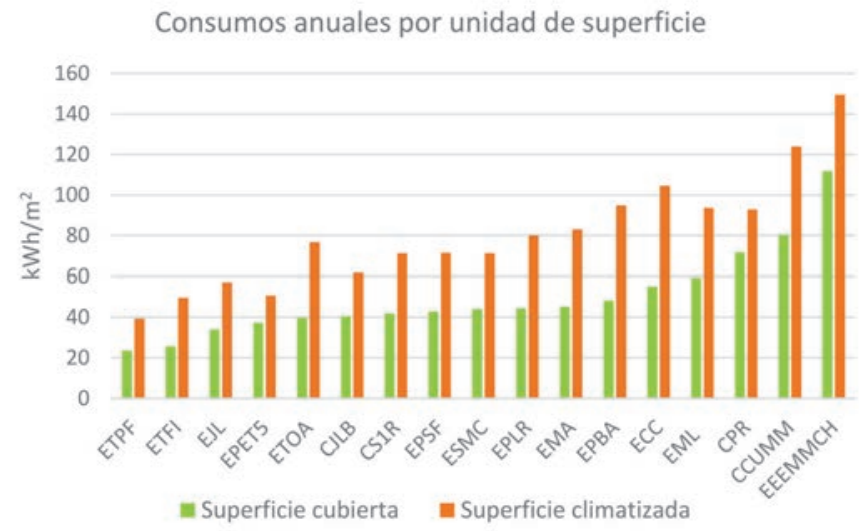

Figura 5: Consumos energéticos anuales por $\mathrm{m}^{2}$ de superficie cubierta y climatizada. Fuente: Elaboración de las autoras.

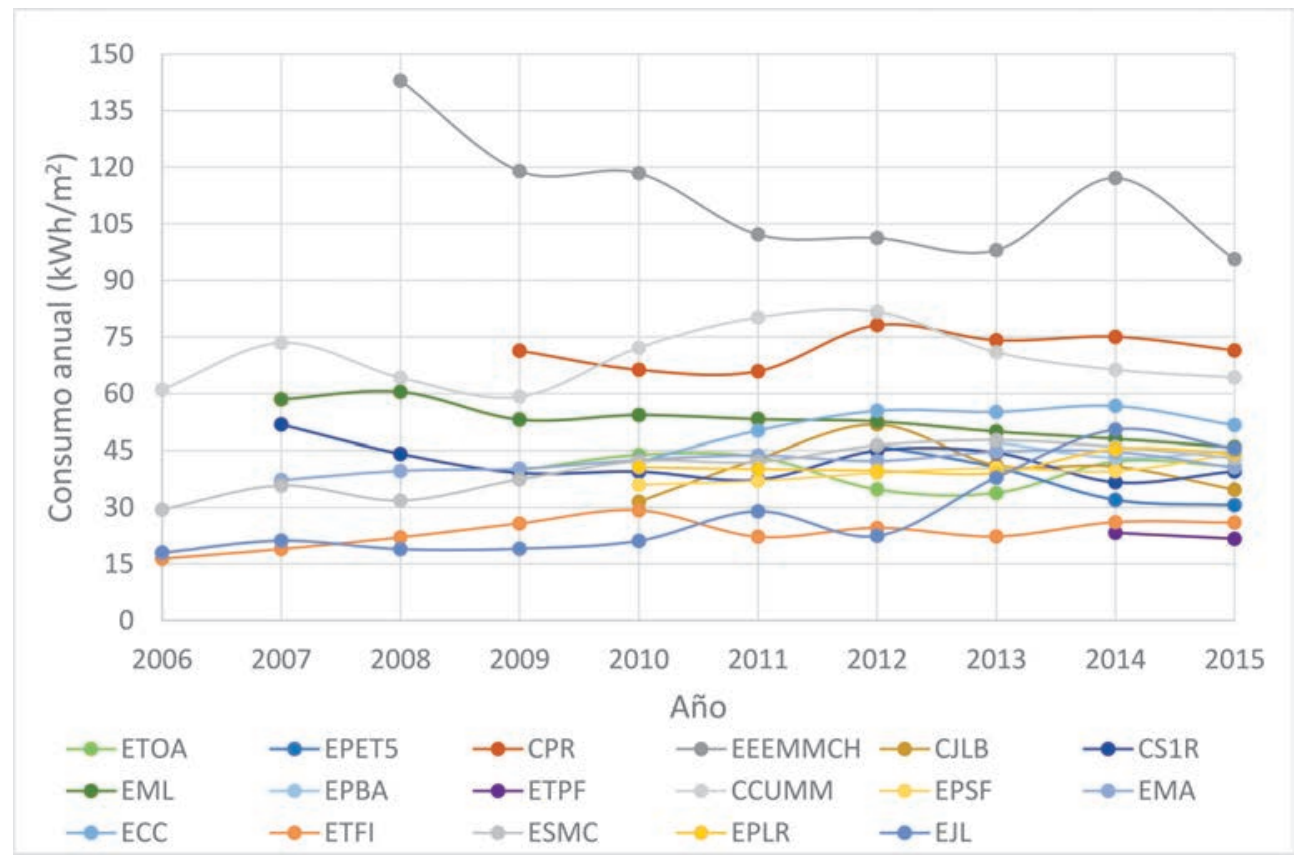

Figura 6: Variación temporal de consumos energéticos anuales por unidad de superficie cubierta. Fuente: Elaboración de las autoras.

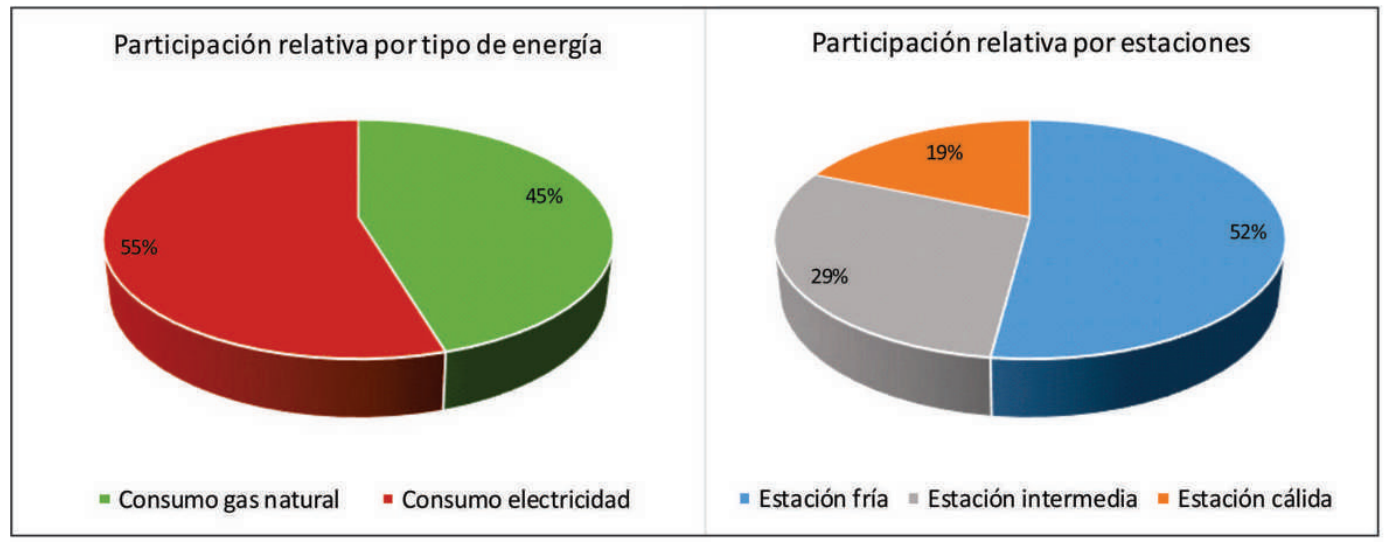

Figura 7: Participación relativa de consumos energéticos anuales promedio (2014 y 2015). Fuente: Elaboración de las autoras. 
Consumo de energía eléctrica por estaciones

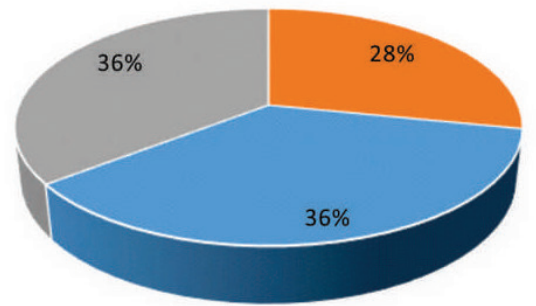

" Estación cálida "Estación fría " Estación intermedia
Consumo de gas natural por estaciones

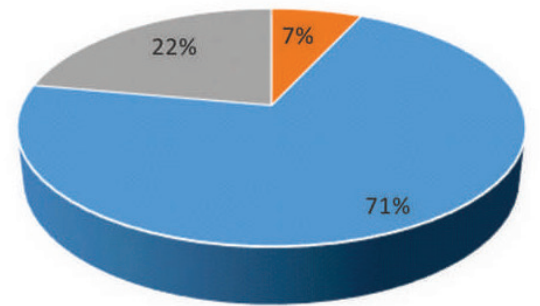

- Estación cálida "Estación fría "Estación intermedia

Figura 8: Consumo de energía eléctrica y gas natural por período estacional. Elaboración de las autoras.

unidad de superficie cubierta, registrados en un período comprendido entre 2006 y 2015. En la Figura 6, se advierte que la escuela EEMMCH ha presentado onsumos elevados desde el comienzo de su actividad en el año 2008; en cambio, el CCUMM, perteneciente a la tipología del Plan Quinquenal, fluctúa entre valores de $59,14 \mathrm{kWh} / \mathrm{m}^{2}$, en el año 2009 , y $81,65 \mathrm{kWh} / \mathrm{m}^{2}$, en 2012 , disputando la segunda posición de mayores consumos con el CPR (PN700), cuyas variaciones van desde los $65,91 \mathrm{kWh} / \mathrm{m}^{2}$, en 2011 , a los $78,1 \mathrm{kWh} / \mathrm{m}^{2}$, en 2012 . Los menores consumos durante toda la instancia de análisis los registran las escuelas ETPF y ETFI.

Por otro lado, se indaga en la participación relativa de los distintos tipos de energía en el total promedio consumido durante 2014 y 2015. Al año calendario se lo divide en tres períodos estacionales, con el propósito de analizar la incidencia de la electricidad y del gas en cada uno de ellos. El período frío se encuentra integrado por los meses de mayo, junio, julio y agosto; el cálido por noviembre, diciembre, enero y febrero, y las estaciones intermedias conformadas por marzo, abril, septiembre y octubre.

La Figura 7 (izquierda) indica que el consumo de electricidad representa un 55\% del total anual promedio. Respecto al consumo total de energía, la consumida en invierno presenta el mayor coeficiente de variabilidad ${ }^{1}$ entre los casos de estudio $(63,9 \%)$ y también la mayor participación relativa estacional (52\%). La energía total consumida durante el ciclo lectivo (períodos frío e intermedio) representa el $81 \%$ y supera ampliamente a la energía consumida en el verano (19\%), situación que se asocia al receso escolar (Figura 7 derecha).

En cuanto al consumo de energía eléctrica (Figura 8 izquierda), su participación relativa en los meses fríos es igual a la del período intermedio (36\%). El hecho de que exista más consumo de electricidad en las estaciones intermedias que en el verano (28\%), se asocia a una mayor demanda para equipamiento, iluminación y refrigeración de espacios, durante el período de clases. En lo que se refiere al gas natural (Figura 8 derecha), el consumo en el período frío asciende al $71 \%$ y muestra el mayor coeficiente de variación entre los casos de estudio (87\%), pudiendo estar asociado a las variables arquitectónicas, a las condiciones climáticas y/o a los comportamientos de los usuarios.

\section{CONSUMOS $Y$ ARQUITECTÓNICAS}

VARIABLES

En la Figura 9, se observan en distintos gráficos de dispersión, las relaciones entre el Área vidriada al Norte $\left(\mathrm{m}^{2}\right)$ y el consumo total anual, estacional, y por fuente de energía. En la Tabla 1 se detallan los indicadores estadísticos del análisis.

\begin{tabular}{|l|l|l|l|l|}
\hline \multicolumn{2}{|l}{ Consumo de energía } \\
\hline \multirow{3}{*}{ Anual } & Total & 0.386 & 0.0001 & 0.62 \\
\cline { 2 - 5 } & Electricidad & 0.504 & 0.0000 & 0.71 \\
\cline { 2 - 5 } & Gas natural & 0.093 & 0.0779 & 0.31 \\
\hline \multirow{2}{*}{ Estación fría } & Electricidad & 0.542 & 0.0000 & 0.74 \\
\cline { 2 - 5 } & Gas natural & 0.162 & 0.0181 & 0.40 \\
\hline \multirow{2}{*}{ Estación cálida } & Electricidad & 0.311 & 0.0006 & 0.56 \\
\cline { 2 - 5 } & Gas natural & 0.395 & 0.0001 & -0.63 \\
\hline \multirow{2}{*}{$\begin{array}{l}\text { Esta cio n e s s } \\
\text { intermedias }\end{array}$} & Electricidad & 0.526 & 0.0000 & 0.725 \\
\cline { 2 - 5 } & Gas natural & 0.018 & 0.4421 & -0.136 \\
\hline
\end{tabular}

Tabla 1: Indicadores estadísticos del análisis de Área vidriada al Norte v/s Consumo de energía. Fuente: Elaboración de las autoras.

[1] El coeficiente de variación es la relación entre la desviación estándar y el promedio *100; donde la desviación típica o estándar es la medida de dispersión paramétrica igual a la raíz cuadrada de la varianza o de la media del cuadrado de las desviaciones de los valores de una distribución respecto a su media (Sierra Bravo, 1991:174). 


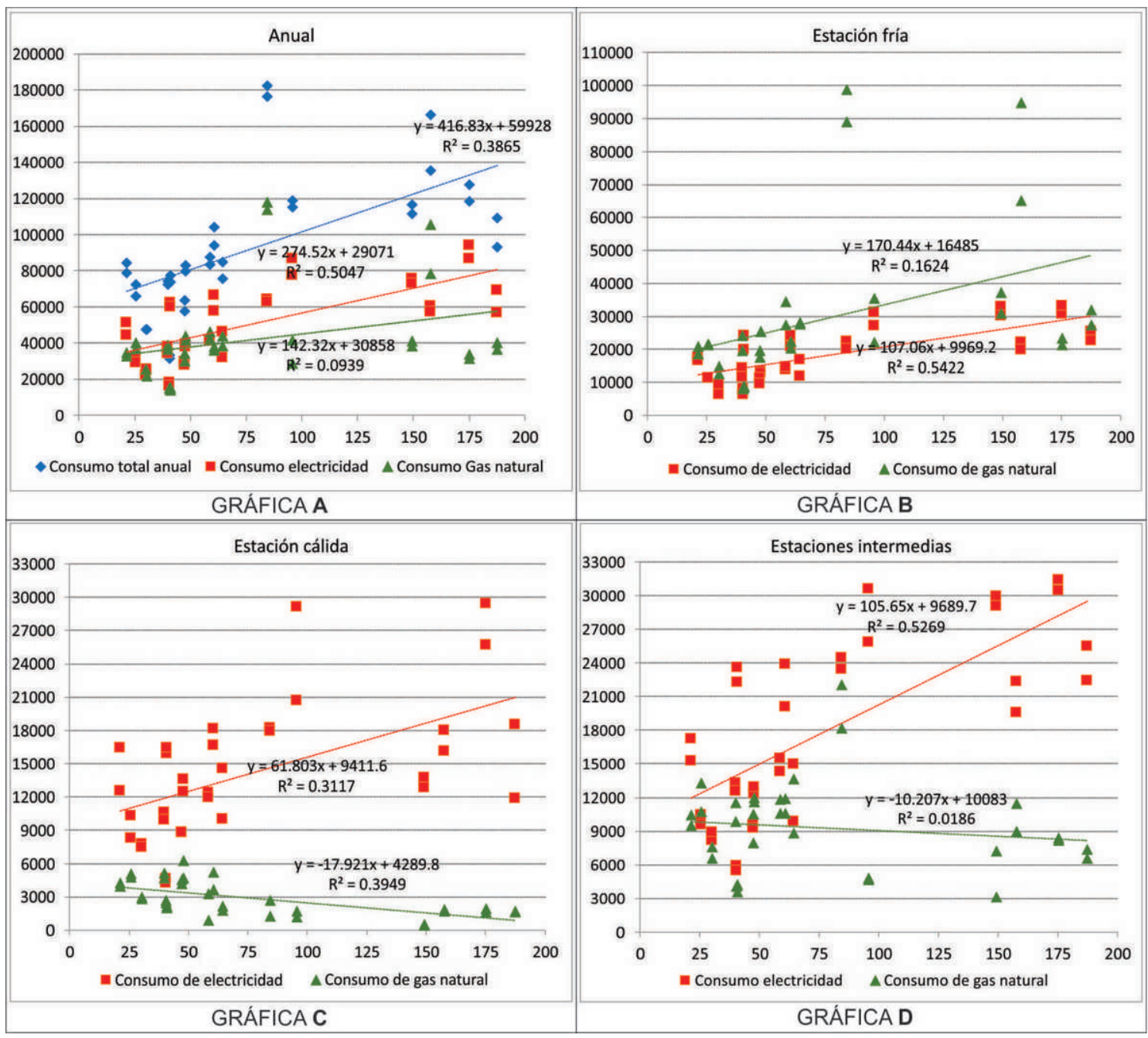

Figura 9: Análisis de regresión simple; Área vidriada al norte $\left(\mathrm{m}^{2}\right)$ en eje X y Consumo de energía (kWh) en eje Y. Grafico A: Consumos anuales. Gráfico B: Estación fría. Gráfico C: Estación cálida. Gráfico D: Estaciones intermedias. Fuente: Elaboración de las autoras.

La Gráfica A, muestra las líneas de regresión y el valor de $\mathrm{R}^{2}$ para los consumos anuales. Para el consumo total y el consumo de electricidad (que absorbe un 55\% del consumo total según la Figura 7 izquierda), los coeficientes de correlación indicarían una relación moderadamente fuerte entre variables con valores de 0,62 y 0,71 , respectivamente, ambos con un Valor - P menor a 0,05. En el caso del gas natural, con un Valor - P de 0,0779, no habría una relación estadísticamente significativa y el coeficiente de correlación $(0,31)$ indicaría una asociación débil entre variables.

Para el período frío y en divergencia con lo esperable ante una variable de diseño que permitiría el ingreso de ganancia solar directa, las líneas de regresión son ascendentes (Figura 9, Gráfica B). Desde el punto de vista estadístico, el coeficiente de correlación para el consumo de electricidad en invierno es de 0,74 y el valor - $\mathrm{P}$ es menor a 0,05, existiendo una relación moderadamente fuerte y estadísticamente significativa entre variables. En el caso del gas natural, el coeficiente de correlación es de 0,40 e indicaría una relación relativamente débil (Tabla
1). Las áreas vidriadas al norte en los edificios estudiados no siempre están libres de obstrucciones, situación que reduciría la efectividad del área colectora. La implantación de especies arbóreas de hojas perennes, la incorporación de carpintería con vidrio repartido y la presencia de parasoles móviles parcialmente cerrados, entorpecen la posibilidad de aprovechar la energía solar para climatizar en invierno los espacios interiores. Además, del aumento del consumo de electricidad para iluminación, por disminución de horas de luz natural, también es posible que su tendencia ascendente se deba a la utilización de equipos de climatización auxiliares alimentados por energía eléctrica, en los sectores administrativos.

En la Figura 9 (Gráfica C), se ilustran las líneas de regresión del comportamiento entre las variables en verano. El consumo de electricidad presenta una tendencia ascendente, con indicadores estadísticos que muestran un Valor - P menor a 0,05 y un coeficiente de correlación de 0,56, que sugiere una relación moderada entre las variables (Tabla 1). Es posible que un diseño inadecuado 
de elementos de sombra para el período estival genere el calentamiento de las áreas vidriadas en determinadas horas y condicione el requerimiento de mayor carga de enfriamiento.

El comportamiento del consumo de energía en las estaciones intermedias se ve representada en la Figura 9 (Gráfica D). En cuanto a la electricidad, se distingue una relación estadísticamente significativa (Valor - $\mathrm{P}$ menor a 0,05$)$ y moderadamente fuerte entre variables, con un coeficiente de correlación de 0,725. Para el gas natural, la relación es negativa, mostrando que a mayor área disminuye el consumo, sin embargo, esta relación es débil (coeficiente de correlación $-0,136$ ) y no es estadísticamente significativa (Valor - $\mathrm{P}=0,4421$ ). Las estaciones intermedias se caracterizan por la presencia de anomalías térmicas, muy marcadas en el caso de San Juan, que presenta días fríos o templados y otros con elevadas temperaturas. De este modo, es posible inferir que el edificio requiere mayor carga auxiliar de refrigeración ante el aumento de temperatura del área vidriada, producto de un diseño adecuado de los elementos de sombra respectivos.

Por último, se relacionan los consumos energéticos con el $\mathrm{K}$ global $\left(\mathrm{W} / \mathrm{m}^{2} .{ }^{\circ} \mathrm{C}\right)$, que se refiere a la tecnología constructiva de la envolvente edilicia (Figura 10). En la Tabla 2, se exponen los indicadores estadísticos del análisis de regresión simple.

\begin{tabular}{|l|l|l|l|l|}
\hline \multicolumn{2}{|l|}{ Consumo de energía } & $R 2$ & Valor-P & $\begin{array}{l}\text { Co eficiente } \\
\text { de correlación }\end{array}$ \\
\hline \multirow{4}{*}{ Anual } & Total & 0.277 & 0.0014 & 0.53 \\
\cline { 2 - 5 } & Electricidad & 0.438 & 0.0000 & 0.66 \\
\cline { 2 - 5 } & Gas Natural & 0.043 & 0.2332 & 0.21 \\
\hline \multirow{2}{*}{ Estación fría } & Electricidad & 0.438 & 0.0000 & 0.66 \\
\cline { 2 - 5 } & Gas Natural & 0.094 & 0.0766 & 0.31 \\
\hline \multirow{2}{*}{ Estación cálida } & Electricidad & 0.341 & 0.0003 & 0.58 \\
\cline { 2 - 5 } & Gas Natural & 0.365 & 0.0002 & -0.60 \\
\hline \multirow{2}{*}{$\begin{array}{l}\text { Es t a cion e s } \\
\text { intermedias }\end{array}$} & Electricidad & 0.434 & 0.0000 & 0.66 \\
\cline { 2 - 5 } & Gas Natural & 0.042 & 0.2426 & -0.21 \\
\hline
\end{tabular}

Tabla 2. Indicadores estadísticos del análisis de K global v/s consumo de energía. Fuente: Elaboración propia.

En la Gráfica A de la Figura 10, se observan las líneas de regresión y el valor de $\mathrm{R}^{2}$ de la relación con el consumo de energía anual. Los resultados evidencian que, para el consumo total de energía y el de electricidad, los coeficientes de correlación indicarían una relación moderada entre variables, con valores de 0,53 y 0,662, respectivamente. En ambos casos, el Valor - $\mathrm{P}$ es menor a 0,05 , dándose una relación estadísticamente significativa con un nivel de confianza del 95\%. En el caso del gas natural, no habría relación entre variables (coeficiente de correlación de 0,21).

Las líneas de regresión del análisis durante el período invernal se ven representadas en la Gráfica B (Figura 10). De acuerdo a los indicadores estadísticos obtenidos (Tabla 2), el coeficiente de correlación para el consumo de electricidad es de 0,66 , lo cual sugiere una relación moderadamente fuerte y estadísticamente significativa (Valor - P menor a 0,05) entre variables. El coeficiente de correlación en el caso del gas natural es de 0,094 e indicaría una relación relativamente débil, con un Valor - $P$ superior a 0,05. Los resultados muestran que el consumo de electricidad aumenta considerablemente con la disminución de la resistencia térmica de la envolvente, lo cual podría indicar, para algunos casos, la necesidad de emplear equipamiento eléctrico auxiliar para calefacción en espacios administrativos.

La Figura 10 (Gráfico C) señala la relación entre las variables en verano, con líneas de regresión ascendentes en el consumo de electricidad, cuyos indicadores estadísticos (Tabla 2) revelan un Valor - $\mathrm{P}$ menor a 0,05 y un coeficiente de correlación de 0,58 , que sugiere una relación moderada. Es notorio el incremento del consumo eléctrico a medida que aumentan los valores de K global, lo cual podría connotar que, a mayor intercambio de calor con el exterior, a través de un envolvente edilicia con valores de resistencias térmicas bajas y coeficientes de absorción altos en las cubiertas de tejas coloniales (PQ) o con revestimientos de baldosas (PN700), se produce un aumento en el requerimiento de energía para enfriamiento.

El comportamiento del consumo de energía eléctrica en estaciones intermedias (Figura 10, Gráfica D) denota una relación estadísticamente significativa (Valor - $\mathrm{P}$ menor a $0,05)$ y moderadamente fuerte entre las variables, con un coeficiente de correlación de 0,66, que podría indicar la utilización equipos de refrescamiento, en determinados momentos de la jornada de clases, para un período que se caracterizan por grandes amplitudes térmicas diarias y estacionarias. En cuanto al gas natural, la tendencia es negativa con una relación débil (coeficiente de correlación $-0,206$; valor $-\mathrm{P}=0,4421$ ).

\section{CONCLUSIONES}

El trabajo llevado a cabo permitió alcanzar el objetivo planteado. Los resultados obtenidos generan un significativo aporte al estado del conocimiento, proporcionando datos referidos a los consumos energéticos de edificios escolares del Área Metropolitana de San Juan, de dos tipologías representativas. A partir del estudio, se reconoce un rango de consumo energético anual comprendido entre 23,56 $\mathrm{kWh} / \mathrm{m}^{2}$ y $80,33 \mathrm{kWh} / \mathrm{m}^{2}$ de superficie cubierta, para las escuelas del Plan Quinquenal, y entre $37,34 \mathrm{kWh} / \mathrm{m}^{2}$ y 


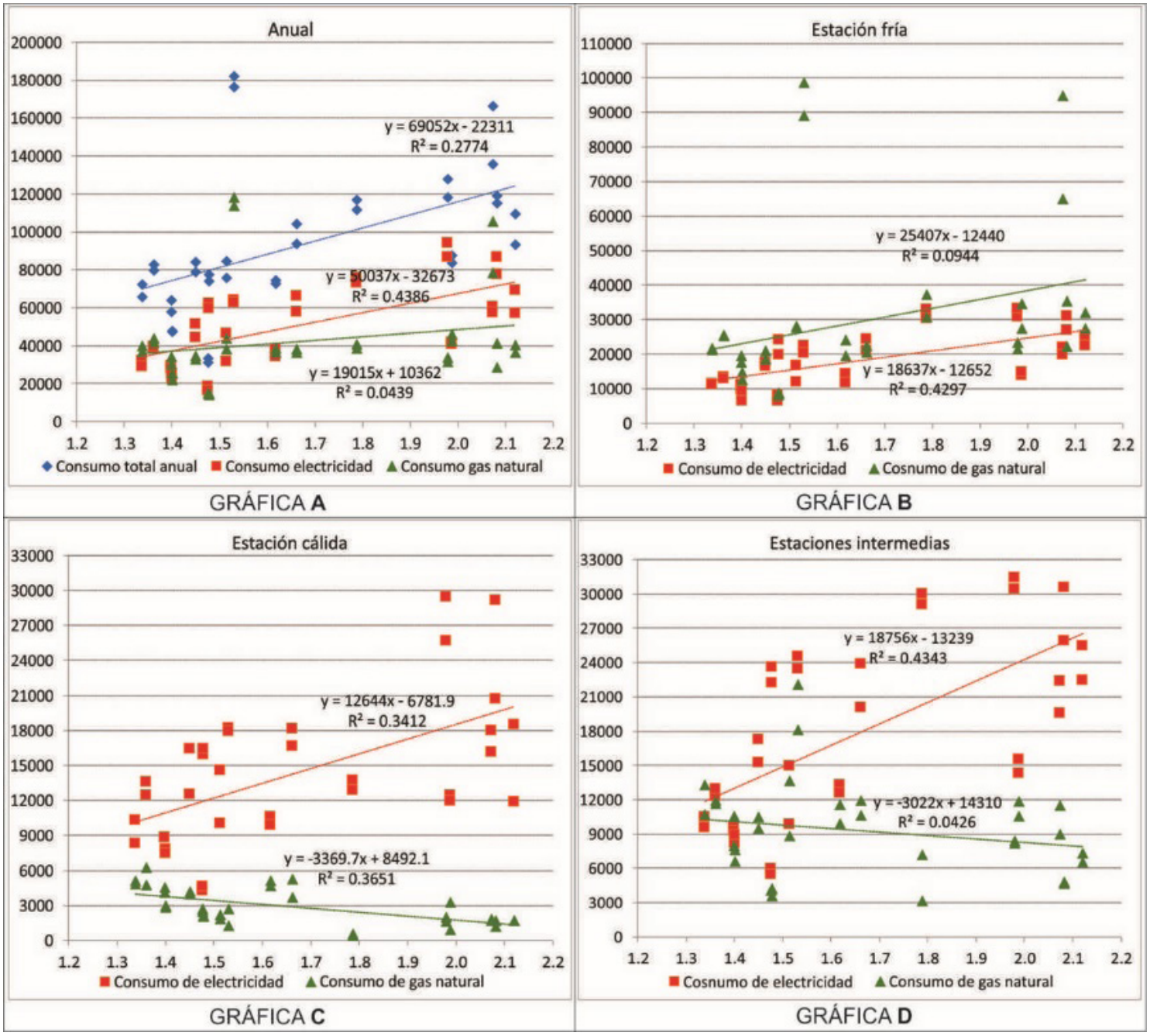

Figura 10: Análisis de regresión simple; K global (W/m².K) en eje X y Consumo de energía (kWh) en eje Y. Gráfico A: Conusmos anuales. Gráfico B: Estación fría. Gráfico C: Estación cálida. Gráfico D: Estaciones intermedias. Fuente: Elaboración propia.

$111,82 \mathrm{kWh} / \mathrm{m}^{2}$, para las que integran el Programa Nacional 700 escuelas. La amplitud de los márgenes de consumo energético en las distintas UA, podrían estar condicionadas por el estado de conservación de las infraestructuras edilicias, las horas y días de uso, y/o los comportamientos de los usuarios y responsables de la operación de los edificios.

La disponibilidad de información de energía consumida condicionó el acotamiento de la muestra a aquellas escuelas con datos ininterrumpidos durante 2014 y 2015. Los resultados revelaron que la electricidad es la más utilizada anualmente, con una participación del 55\%, dado que sirve para abastecer la iluminación diurna y nocturna, el funcionamiento de equipamiento (heladeras, freezer, computadoras, impresoras), el refrescamiento de ambientes y, en algunos casos, la calefacción auxiliar en sectores docentes y administrativos. En cuanto al consumo energético estacional, los mayores registros se producen en invierno con un $52 \%$, con una demanda de gas natural que asciende al $71 \%$. El consumo de energía por unidad de superficie promedio para los casos de estudio, es de 21,80 $\mathrm{kWh} / \mathrm{m}^{2}$ (superficie cubierta) y $35,50 \mathrm{kWh} / \mathrm{m}^{2}$ (superficie climatizada), para el gas natural, y de $26,24 \mathrm{kWh} / \mathrm{m}^{2}$ y 42,73 $\mathrm{kWh} / \mathrm{m}^{2}$ respectivamente, para la electricidad.

El estudio estadístico permitió concluir que, para la muestra analizada, el Área vidriada al Norte es una variable influyente en los consumos energéticos totales y de electricidad, pero no en los de gas natural (principal fuente energética utilizada para calefacción). Las ganancias solares directas en invierno no serían suficientes para contribuir a la climatización de los espacios interiores debido a posibles obstrucciones, pero afectarían negativamente los ambientes en verano por sobrecalentamiento. Fue posible detectar, en los casos que presentan los mayores consumos por unidad de superficie climatizada (EEMMCH, CCUMM, ECC, EPBA), que gran parte de las aulas y los espacios administrativos se encuentran orientados al Sur o al Este, y las áreas vidriadas al Norte corresponden a circulaciones cerradas o galerías. 
Respecto a la variable $\mathrm{K}$ global, se reconoce una fuerte relación del aumento del consumo eléctrico en los meses de verano y estaciones intermedias ante envolventes menos eficientes; factor que podría indicar la mayor utilización de equipos auxiliares para refrigeración de espacios, como causa de una envolvente edilicia que presenta intercambios de calor en horarios del cursado de clases.

Los análisis realizados con las variables arquitectónicas permitieron ratificar la importancia del diseño de la envolvente y su asociación con el consumo de energía. Dichas variables exhiben un fuerte potencial de mejoramiento: un cambio que apunte a incrementar la eficiencia energética de edificios escolares, preservando y/u optimizando las condiciones de confort térmico y lumínico.

\section{AGRADECIMIENTOS}

La información presentada forma parte de la Tesis Doctoral de la Mag. Arq. Guillermina Ré, del Doctorado en Arquitectura, de la Universidad de Mendoza. Se agradece a la Dra. Florencia Ricard por su asesoramiento; y a la Universidad Nacional de San Juan, FAUD, por la contribución en el financiamiento de la investigación en el marco del PROJOVI 2018- 2019.

\section{REFERENCIAS BIBLIOGRÁFICAS}

BLASCO LUCAS, Irene. Arquitectura sustentable en hábitat rural de zona árido-sísmica: Aportes teórico-metodológicos. Tesis Doctoral en Arquitectura. Universidad de Mendoza. 2013.

BOUTET, M. Laura; HERNÁNDEZ, Alejandro y JACOBO, Guillermo. Thermo-lighting optimization proposal for school buildings in subtropical hot-humid climates: Monitoring and computer simulation on autumn period, Energy and Buildings, 2016, vol. 128, pp. 785-797.

BOUTET, María Laura. Acondicionamiento higrotérmicolumínico de edificios escolares en zonas urbanas de la región N.E.A. Auditorías energéticas y propuestas de mejoras mediante Diseño Solar Pasivo. Tesis Doctoral. Universidad Nacional de Salta. Facultad de Ciencias Exactas. Doctorado en Ciencias- Área Energías Renovables, 2017

CZAJKOWSKI, Jorge D. Análisis y modelización energéticoambiental de la edilicia urbana basado en técnicas de auditoría y procedimientos estadísticos multivariados. Desarrollo de herramientas de diagnóstico y simulación. Tesis Doctoral. Departamento Construcciones, Facultad de Ingeniería, UNLP, 2009

DROUTSAA, K.G.; KONTOYIANNIDISA, S.; DASCALAKIA, E.G. and BALARASA, C.A. Benchmarking energy use of existing Hellenic Non-Residential buildings. En: BIKAS, Dimitrios (ed.). International Conference on Sustainable Synergies from Buildings to the Urban Scale, SBE16. Science Direct. Procedia Environmental Sciences, vol. 38, 2017, pp. 713-720
FILIPPÍN, Celina. Thermal response of solar and conventional school buildings to design- and human-driven factors, Renewable Energy, 2005, vol. 30, n³, pp. 353 - 376.

GIULIANO, Gabriela y GARZÓN Beatriz. Eficiencia térmicaenergética y adecuación bioambiental en escuela rural de Santiago del Estero- Argentina. En: Actas ASADES, vol. 5, 2017, pp. $05.143-05.153$

GONZALO, Guillermo; NOTA, Viviana; LEDESMA, Sara y MARTÍNEZ, Cecilia. Programa para el cálculo de Transmitancia térmica ( $K$ medio ponderado), retardo y amortiguamiento. CEEMA: Centro de Estudios Energía y Medio Ambiente. IAA. FAU. UNT, 2000.

LEDESMA, Sara; CISTERNA, Susana; NOTA, Viviana; MARTÍNEZ, Cecilia; QUIÑONES, Graciela; MÁRQUEZ VEGA, Gabriela; LLABRA, Cristina; GONZALO Guillermo; MOSTAJO, María; RAMOS, Mariano y VILLA, Cyntia. Caracterización del sector edilicio educativo de nivel primario de la provincia de Tucumán y evaluación de consumos energéticos en casos de estudio. En: Actas ASADES, vol. 4, 2016, pp. 05.83 - 05.94.

MAZZOCCO, María Pía; FILIPPÍN, Celina y FLORES LARSEN, Silvia. Impacto potencial del cambio climático en una escuela solar en Argentina, Energías Renovables y Medio Ambiente, 2017, vol. 39, pp. 49-60.

MELCHIORI, Mariana; SAN JUAN Gustavo y DÍSCOLI, Carlos. Comparación del comportamiento energético en dos establecimientos educativos: Campinas (Brasil) y La Plata (Argentina), Revista Estudios del Hábitat, 2014, n 12, pp. 63-81. MICHAUX, M. Celina. Estudio tipológico y simulación del comportamiento térmico de edificios escolares en la ciudad de San Juan. Informe Final de Beca CICITCA, Estudiantes Avanzados. UNSJ, 2014.

MOHAMED M., Ouf, MOHAMED H., Issa. Energy consumption analysis of school buildings in Manitoba, Canada, International Journal of Sustainable Built Environment. 2017, vol. 6, n² 2, pp. 359-371.

MUÑOZ, Natalia; MARINO, Beatriz y THOMAS, Luis. Caracterización térmica de edificios aplicando el modelo de regresión lineal múltiple. En: Actas ASADES, vol. 4, 2016, pp. $05.31-05.42$

Programa Nacional 700 Escuelas. Manual de proyecto. Argentina: Ministerio de Educación, Ciencia y Tecnología Nación. Ministerio de Planificación Federal, Inversión Pública y Servicios, 2004.

RAATIKAINEN, Mika; SKÖNA, Jukka; LEIVISKÄB, Kauko y KOLEHMAINENA, Mikko. Intelligent analysis of energy consumption in school buildings, Applied Energy, 2016, vol. 165, pp. 416-429.

RÉ, Guillermina; BLASCO LUCAS, Irene y FILIPPÍN, Celina. Evaluación higrotérmica y energética de un edificio escolar perteneciente al Programa Nacional 700 Escuelas, en el Área Metropolitana de San Juan, Argentina, Revista Hábitat Sustentable, 2016, vol. 6, $n^{\circ} 2$, pp. 40-51.

RÉ, Guillermina; BLASCO LUCAS, Irene y FILIPPÍN, Celina. Comportamiento higrotérmico y energético en período estival de un edificio escolar típico en la ciudad de San Juan, Argentina, Revista Estudios del Hábitat, 2017, vol. 15, n² 2, pp.1-12. 
ROSPI, Gianluca; CARDINALE, Nicola; INTINI, Francesca y CARDINALE, Tiziana. Analysis of energy consumption of different typologies of school buildings in the city of Matera (Southern Italy), Science Direct. Energy Procedia, 2015, vol. 82, pp. 512518.

SECRETARIA DE ENERGÍA DE LA NACIÓN. Balances energéticos 2010 [en línea]. [Consultado 20 junio 2018]. Disponible en: www. energía3.mecon.gov.ar. 2012

SIERRA BRAVO, Restituto. Diccionario práctico de estadística. Madrid: Editorial Paraninfo, 1991.

TREBILCOCK KELLY, Maureen; SOTO MUÑOZ, Jaime; FIGUEROA SAN MARTIN, Rodrigo; PIDERIT-MORENO, Beatriz. Metodología para el diseño de edificios educacionales confortables y resilientes, Revista AUS, 2016, n²0, pp. 70-76.

WALTER, Érika; AGÜERO, Matías; WATKINS, María Gabriela y MANSILLA, Gabriela. Escuela bioclimática y sustentable, Secundaria № 39, Villa de Antofagasta de la Sierra, Catamarca. En: Acta del I Encuentro Nacional sobre Ciudad, Arquitectura y Construcción Sustentable (La Plata), 2016, pp. 131-141.

WOO, Kim Tae; GUK, Lee Kang; HWA, Hong Won. Energy consumption characteristics of the elementary schools in South Korea, Energy and Buildings, 2012, vol. 54, pp. 480- 489.

YACANTE, María Irene; GUILLÉN RODRÍGUEZ, Sandra; BUSTOS, Pamela; HEREDIA, Sergio y CRUZ INTROÍNI, Melina. Identificación de vulnerabilidad sísmica no estructural y funcional en edificios escolares del Gran San Juan como aporte a la proyectación. En: Actas del XXI ARQUISUR. San Juan, Argentina, 2017, Eje 2- Art. 8.

YOSHINO Hiroshi; HONG Tianzhen y NORD Natasa. IEA EBC Annex 53: Total energy use in buildings. Analysis and evaluation methods, Energy and Buildings, 2017, vol. 152, pp. 124-136. 